\title{
¿QUÉ TIPO DE DATOS RECOLECTAN LOS DIRECTORES? CONSECUENCIAS PARA LA ELABORACIÓN DE PLANES DE MEJORA
}

\author{
What type of data collect school principals? \\ Consequences for educational development plans
}

\author{
MARTA QUIROGA 1 \\ FELIPE ARAVENA ${ }^{2}$ \\ Pontificia Universidad Católica de Valparaíso. Chile. \\ Correspondencia: marta.quiroga@pucv.cl, felipe.aravena@pucv.cl
}

Recibido: $15-12-2016$

Revisado: 14-03-2017

Aceptado: 11-10-2017

Resumen: El propósito de esta investigación es analizar la recolección de datos que realizan los directivos docentes y cómo influye esta información en el diseño de planes de mejoramiento educativo. Mediante un estudio cuantitativo de carácter exploratorio-descriptivo, se tradujo y validó una encuesta de autoevaluación de recolección de datos que se aplicó a un grupo de 24 directivos. Los resultados muestran que los directores recolectan preferentemente datos sobre el comportamiento de los alumnos, sobre la relación escuela-familia y sobre las prácticas de enseñanza de los profesores. Se concluye que los directores cuentan con pocos datos del núcleo pedagógico, lo que impide que los planes de mejoramiento logren cambios o mejoras en el aula.

Palabras clave: liderazgo escolar; toma de decisiones; datos.

\begin{abstract}
The purpose of this research is to analyze the collection of data by principals and how this information influences the design of educational improvement plans. Through a quantitative exploratory-descriptive study, a self-assessment survey of data collection was translated and validated and applied to a group of 24 principals. The results show that principals preferentially collect data on student behaviour, school-family relations and teachers' teaching practices. It is concluded that the principals have limited data on the pedagogical nucleus, which prevents the improvement plans from achieving changes or improvements in the classroom.
\end{abstract}

Keywords: educational leadership; decision-making; data.

\footnotetext{
${ }^{1}$ Doctora en Educación de la Universidad de Sevilla (España). Académica en la Escuela de Pedagogía de la Pontificia Universidad Católica de Valparaíso (Chile). Líneas de trabajo: formación docente inicial y formación profesional de directores escolares.

${ }^{2}$ Magíster en Liderazgo Educativo en The University of Western Australia (Australia). Académico en la Escuela de Pedagogía de la Pontificia Universidad Católica de Valparaíso (Chile). Líneas de trabajo: liderazgo educativo y formación profesional de directores escolares.
} 


\section{INTRODUCCIÓN}

Elaborar e implementar planes de mejoramiento educativo (PME) es una estrategia de cambio planificado que actualmente se implementa en diversos países latinoamericanos tales como Ecuador en el 2012, Chile en el año 2014 y Perú en el 2013, entre otros. Para su elaboración e implementación se requieren procesos de recolección de datos. Inicialmente estos se recolectan para realizar la autoevaluación institucional. Se aplican diferentes instrumentos que, sistematizados, permiten obtener información, la que es utilizada para orientar el diseño del PME. Posteriormente, en la fase de implementación, se realizan las acciones y nuevamente se recolectan datos y evidencias que permitan conocer el grado de ejecución de las actividades y el nivel de logro de los objetivos propuestos. Por ello, las actividades de recolección de información son acciones que se realizan permanentemente en el contexto de PME, de aquí que sea importante indagar sobre qué tipos de datos recolectan los directivos y sus implicancias para la elaboración de planes de mejora institucional. Para lograr este objetivo se han formulado las siguientes preguntas de investigación: 1) ¿Qué datos recolectan los directivos docentes? y 2) ¿Cómo estos datos se relacionan con las áreas de PME?

Para responder estas preguntas se elaboró un marco de referencia que se apoya en cuatro campos de investigación, que posteriormente son relacionados con las áreas y dimensiones de los PME en Chile. En primer lugar, se discute el rol de los directivos docentes en la mejora de aprendizaje de los alumnos (Anderson y Bennet, 2003; Fullan, 2009; Harris, 2009; Heifetz, 1994; Robinson, Lloyd y Rowe, 2008); en segundo lugar el rol de los datos en la toma de decisiones en directivos docentes (Cooley et al., 2006; Knapp, Swinnerton, Copland y Monpas-Huber, 2006; Shen et al., 2007; Shen y Cooley, 2008; Schildkamp y Kuiper, 2010; Honig y Coburn, 2008; Marsh y Farrell, 2015; Seyal, 2015; Lynch, Smith, Provost y Madden, 2016; Datnow y Hubbard, 2016); en tercer lugar se analiza la tipología de datos y diversas posibilidades de realizar análisis múltiples (Bernhardt, 2003) y, en cuarto lugar, se caracterizan las áreas de planificación de los PME en Chile.

La información fue recopilada a través de un cuestionario de autoevaluación de recolección de datos para directivos docentes, el que fue traducido, adaptado y validado. El instrumento fue creado por Shen et al. (2012) y se apoya en el metaanálisis de 70 estudios realizado por Marzano, Waters y McNulty (2003), en el que se identifican 11 estrategias que generaron mejoras sustanciales en los aprendizajes de los estudiantes. El cuestionario consta de 42 preguntas, las que se agrupan en tres factores: escuela, profesores y alumnos.

\section{Los directores escolares y su rol}

En los últimos veinte años el rol de los directores escolares ha cambiado desde la administración a la gestión y, actualmente, al liderazgo centrado en el aprendizaje. Abundante bibliografía concluye que las prácticas de los directores influencian positivamente las prácticas de los profesores y, en consecuencia, impactan en la 
calidad de los aprendizajes de los estudiantes (Bolívar, 2012; Elmore, 2010; Leithwood et al., 2008; Swaffield y Macbeath, 2009). Para liderar una organización centrada en el aprendizaje se necesita un equipo comprometido que comparta una visión, metas claras y sentido de propósito (Hargreaves y Fink, 2006; Fullan, 2007). Un líder necesita de equipos para liderar y direccionar la organización hacia los objetivos de aprendizaje, para ello animar y alentar las relaciones sociales, la colaboración profesional y el compromiso de los docentes es una de las tareas primordiales que deben sortear los líderes escolares (Leithwood et al., 2008; Fullan, 2007; Harris, 2009; Heifetz, 1994).

Leithwood y Riehl (2005) desarrollaron un estudio en el que describen diversas áreas de liderazgo de los directores efectivos y las prácticas asociadas (Tabla 1). Estas son: establecer dirección de futuro, desarrollar al personal, rediseñar la organización y gestionar los programas de enseñanza y aprendizaje. Cada una de estas dimensiones se encuentra asociada a prácticas específicas, las que promueven motivación a los docentes, desarrollan nuevas habilidades o capacidades y generan condiciones de trabajo.

Tabla 1. Prácticas claves para el liderazgo efectivo

\begin{tabular}{|c|c|}
\hline Categorías & Prácticas de liderazgo \\
\hline \multirow[t]{3}{*}{ Mostrar dirección de futuro } & Visión (construcción de una visión compartida) \\
\hline & Objetivos (fomentar la aceptación de objetivos) \\
\hline & Altas expectativas \\
\hline \multirow[t]{4}{*}{ Desarrollar personas } & Atención y apoyo individual \\
\hline & Atención y apoyo intelectual \\
\hline & $\begin{array}{l}\text { Modelamiento (interacción permanente y } \\
\text { visibilidad con los alumnos y estudiantes) }\end{array}$ \\
\hline & $\begin{array}{l}\text { Conectar a su escuela con su entorno } \\
\text { (y las oportunidades) }\end{array}$ \\
\hline \multirow[t]{4}{*}{ Rediseñar la organización } & Construir una cultura colaborativa \\
\hline & $\begin{array}{l}\text { Estructurar una organización que facilite } \\
\text { el trabajo }\end{array}$ \\
\hline & $\begin{array}{l}\text { Crear una relación productiva con la familia } \\
\text { y comunidad }\end{array}$ \\
\hline & $\begin{array}{l}\text { Conectar a la escuela con su entorno } \\
\text { (y sus oportunidades) }\end{array}$ \\
\hline \multirow{4}{*}{$\begin{array}{l}\text { Gestionar la instrucción (enseñanza y } \\
\text { aprendizaje) en la escuela }\end{array}$} & Dotación de personal \\
\hline & $\begin{array}{l}\text { Proveer apoyo técnico a los docentes } \\
\text { (supervisión, evaluación y coordinación) }\end{array}$ \\
\hline & $\begin{array}{l}\text { Monitoreo (de las prácticas docentes } \\
\text { y de los aprendizajes) }\end{array}$ \\
\hline & $\begin{array}{l}\text { Evitar distracciones de lo que no es el centro } \\
\text { de su trabajo }\end{array}$ \\
\hline
\end{tabular}


De acuerdo con la Tabla 1, la categoría "mostrar dirección de futuro" se encuentra asociada a la motivación de los profesores; la de "desarrollar personas" se asocia al desarrollo de capacidades profesionales; y las de "rediseñar la organización" y "gestionar la instrucción" se asocian a la generación de condiciones de trabajo.

Los directivos que despliegan estas prácticas de forma efectiva cuentan con datos e información relevante. El estudio de Schildkamp y Kuiper (2010) identificó 5 razones por las cuales los directivos docentes recolectan datos: monitorear el progreso e identificar las áreas de necesidad; desarrollar y planificar políticas; evaluar el desempeño de los maestros; apoyar las conversaciones con los maestros y satisfacer las demandas de rendición de cuentas. Todas ellas vinculadas con las prácticas de gestionar la instrucción. En línea con la evidencia planteada por Leithwood y Riehl (2005).

Desarrollar prácticas de usos de datos en las organizaciones requiere conocimientos, habilidades y creencias favorables sobre su uso. Estos se modelan especialmente en las comunidades profesionales, ya que los profesores tienen variadas creencias, experiencias y conocimientos sobre el uso de datos. El estudio de Datnow y Hubbard (2016) indica que para tener más éxito la formación debe orientarse a las creencias de los maestros y el uso de datos debe estar desvinculado de demandas de rendición de cuentas externas. Lo anterior puede ser complementado por el estudio elaborado por Marsh y Farrell (2015) que, desde un marco de aprendizaje sociocultural, caracterizó el apoyo de entrenadores externos en el uso de datos en establecimientos escolares, concluyendo que los líderes escolares requieren animar, apoyar el desarrollo de comunidades profesionales y construir en ellas relaciones interpersonales y de confianza para que los educadores se conozcan y dialoguen confidencialmente sobre los datos. Otro elemento importante es el uso de Tecnologías de la información y Comunicación (TIC) en el análisis de datos y liderazgo; el estudio de Seyal (2015) identificó una relación positiva entre el uso de TIC para análisis de datos y el liderazgo transformacional. Sin embargo, la evidencia empírica sobre esta relación aún es escasa.

\section{Toma de decisiones informadas por datos}

Una actividad fundamental del director es la toma de decisiones, es decir, decidir entre varias opciones de solución frente a un problema. Peter Drucker (2002) se pregunta sobre los pasos a seguir para tomar decisiones y sobre la naturaleza de los problemas e identifica cuatro tipos de problemas: los auténticamente genéricos, que se presentan comúnmente en la organización y en algunas oportunidades representan síntomas de un nudo crítico; los verdaderamente genéricos, que tratan sobre acontecimientos singulares, es decir, no son repetitivos en una organización específica pero en el medio son comunes; los auténticamente excepcionales o únicos, que son situaciones de decisión en las cuales el problema a resolver es irrepetible o difícilmente repetible; y finalmente los que son un nuevo problema genérico. 
La habilidad que poseen los líderes escolares para identificar la naturaleza del problema es clave para tomar decisiones inteligentes, conscientes e intencionales (Drucker, 2002; Seyal, 2015).

Con relación a los pasos para solucionar problemas se han identificado seis: clasificar el problema, definir el problema, identificar las condiciones que tiene que satisfacer la respuesta al problema, decidir sobre lo que es correcto en lugar de lo que es aceptable, identificar las acciones que se han de llevar a cabo para implementar la decisión y, comprobar la validez y eficacia de la decisión en relación con el estado actual de los hechos. Cada uno de estos pasos posee una complejidad que le es propia. En este artículo se analiza el segundo paso, es decir, la definición del problema.

La discusión bibliográfica sobre cómo los directivos docentes usan los datos para tomar decisiones ha evolucionado desde "guiados por datos" hacia "basados en datos" y, actualmente, a "informados por datos" (Cooley et al., 2006; Knapp, Swinnerton, Copland y Monpas-Huber, 2006; Shen et al., 2007; Shen y Cooley, 2008). Actualmente existe consenso en que los datos informan y no dirigen la toma de decisiones. Esto se debe a que la toma de decisiones está influenciada por diversos elementos: cognitivos, éticos, morales, políticos, jurídicos y contextuales, especialmente en el caso de directores escolares. La toma de decisiones informada por datos requiere de los directivos docentes un grado de preparación para desarrollar o adecuar instrumentos reflexionando sobre la producción de información y el tratamiento estadístico de ellos, dado que existen componentes técnicos y contextuales que pueden limitar su utilización y, como plantean Honig y Coburn (2008), el uso de datos para acciones de mejora genuina puede ser difícil, ya que los datos son a veces ambiguos y no proporcionan directrices claras para la acción (Drucker, 2002).

Desde la perspectiva de la investigación y práctica relacionada con la toma de decisiones informada por datos, se presentan dos problemas. El primero de ellos corresponde a que generalmente los directivos docentes trabajan con datos de resultados, no de procesos. Por ejemplo, analizan las calificaciones de los estudiantes (dato de resultado) y no la frecuencia y calidad de la planificación de los profesores (dato de proceso). El segundo, ya mencionado en el párrafo anterior, es la necesidad de mejorar la formación de los líderes para acceder, generar, gestionar e interpretar datos (Knapp, Swinnerton, Copland y Monpas-Huber, 2006).

\section{Tipología de datos}

Las organizaciones escolares producen muchos datos. Frecuentemente son analizados los resultados de aprendizaje de los alumnos (pruebas estandarizadas) y resultados de eficiencia interna del establecimiento (índices de aprobación, repitencia, entre otros). Si bien no se desconoce la importancia de los primeros, es primordial que los directores y profesores analicen datos de procesos internos, lo que potenciará el trabajo reflexivo de profesores y directores, al mismo tiempo que la autorreflexión 
y la rendición de cuentas internas (Elmore, 2010). Al respecto, Bernhardt (2003) ha desarrollado una propuesta teórica que clasifica los datos en cuatro categorías: demográficos, percepción, proceso y resultados, proponiendo diez tipos de preguntas que se pueden formular, interrelacionándolos. Estas van desde las preguntas de nivel 1, que usan un solo tipo de dato, por ejemplo: ¿cuál es el promedio de quinto básico en Lenguaje?; hasta las preguntas de nivel 10, en que se pide utilizar los cuatro tipos de datos en un tiempo prolongado, por ejemplo: ¿cómo es el rendimiento en los últimos cuatro años en Lenguaje de alumnos que tienen docentes evaluados como destacados en el sistema nacional de evaluación docente, que reciben retroalimentación sobre la planificación de sus clases y que tienen un reporte positivo sobre la calidad de sus clases por parte de sus estudiantes?. Las preguntas van incrementando su complejidad en la medida en que incluyen más tipos de datos y las mediciones incluyen más de un año.

A continuación, describimos cada tipo de datos:

- Datos demográficos: corresponden a las características sociodemográficas de la comunidad educativa. Involucran la caracterización de alumnos, padres y apoderados, personal de apoyo, profesores y directivos docentes. Por ejemplo, nivel educativo de los padres, temáticas de perfeccionamiento de los profesores, entre otros.

- Datos de percepción: indican el grado de valoración que tienen los miembros de la comunidad escolar sobre las acciones e interrelaciones en su interior. Por ejemplo, el grado de satisfacción de los padres sobre las metodologías de enseñanza.

- Datos de proceso: surgen de las actividades de seguimiento y monitoreo de la enseñanza. Por ejemplo, calidad de la docencia a partir de los datos de observación de aula. Es el área en que se agrupan los datos de preparación, implementación curricular, liderazgo directivo y docente.

- Datos de logro: en esta categoría se agrupan los resultados de aprendizaje de los alumnos. Por ejemplo, las calificaciones de asignaturas, los resultados de pruebas estandarizadas. Es decir, son datos cuantitativos sobre las habilidades y manejo de contenidos que han logrado los alumnos.

El análisis múltiple interrelaciona diferentes tipos de datos, potenciando la comprensión sobre los problemas de la organización escolar y permitiendo identificar las causas de ellos. Realizar preguntas que vinculen dos o más tipos de datos implica establecer relaciones complejas, por ejemplo, preguntarse cómo se relacionan los resultados de aprendizajes (resultados) con la opinión de los alumnos sobre la clase (percepción) y la frecuencia de planificación del profesor (proceso). Esta pregunta grafica un análisis múltiple de datos, ya que interrelaciona tres tipos, lo que potencia una comprensión profunda de los desafíos de planificar, implementar y evaluar las acciones del PME. En este sentido, los datos y el análisis de ellos son importantes para los procesos de mejora. 


\section{Planes de mejora (PME)}

Los PME responden a un esfuerzo práctico, sistemático y contextualizado que realiza un establecimiento escolar para mejorar los aprendizajes de los estudiantes a través de la gestión de innovaciones planificadas, implementadas y evaluadas (Canto-Mayo, 2004). Las orientaciones del Ministerio de Educación de Chile para elaborar planes de mejora recomiendan cuatro pasos: autoevaluación, diseño, implementación y evaluación. Estos se incluyen en un ciclo anual, tomando como referencia los datos recogidos en cada una de las acciones implementadas. Los datos y su análisis permiten a los equipos directivos y a las comunidades educativas valorar sus avances y reconocer los desafíos, emprendiendo nuevas acciones. Por ello es importante reconocer qué tipo de datos recolectan los directivos docentes.

Los PME, generalmente, se inician a partir de un proceso de autoevaluación que realiza el establecimiento educacional. En el caso chileno a partir de las orientaciones ministeriales que especifican áreas divididas en dimensiones y expresadas en prácticas específicas (Tabla 2). Se entiende por prácticas los modos de actuar y de relacionarse que las personas despliegan en espacios concretos de acción (Programa de las Naciones Unidas para el Desarrollo, 2009). A continuación, se presentan las áreas, con sus dimensiones y el número de prácticas asociadas.

Tabla 2. Áreas y dimensiones de autoevaluación y elaboración de PME

\begin{tabular}{|l|l|c|}
\hline \multicolumn{1}{|c|}{ Área } & \multicolumn{1}{|c|}{ Dimensión } & N. de prácticas \\
\hline \multirow{4}{*}{ Gestión del currículum } & Gestión pedagógica & 7 \\
\cline { 2 - 3 } & Enseñanza y aprendizaje en el aula & 6 \\
\cline { 2 - 3 } & Apoyo al desarrollo de los estudiantes & 7 \\
\hline \multirow{4}{*}{ Liderazgo } & Liderazgo del sostenedor & 6 \\
\cline { 2 - 3 } & Liderazgo formativo y académico & 7 \\
\cline { 2 - 3 } & Planificación y gestión de resultados & 6 \\
\hline \multirow{3}{*}{ Gonvivencia escolar } & Formación & 6 \\
\cline { 2 - 3 } & Convivencia escolar & 6 \\
\cline { 2 - 3 } & Participación & 6 \\
\hline \multirow{3}{*}{ Gestión de recursos } & Gestión de recursos humanos & 9 \\
\cline { 2 - 3 } & Gestión de recursos financieros y administrativos & 6 \\
\cline { 2 - 3 } & Gestión de recursos educativos & 5 \\
\hline
\end{tabular}

Fuente: Ministerio de Educación de Chile (2014)

El desafío de mejorar los aprendizajes de los estudiantes requiere de la implementación de actividades de seguimiento y monitoreo que produzcan datos que, analizados adecuadamente, provean de información suficiente para valorar el impacto de las acciones del PME. 
Para cerrar esta sección se ha elaborado la Tabla 3, en que se relacionan las estrategias de alto impacto identificadas por Marzano et al. (2003) que son la base del cuestionario de autoevaluación, las dimensiones del PME y la tipología de datos presentada.

Tabla 3. Relación entre estrategias de alto impacto, dimensiones del PME y tipología de datos

\begin{tabular}{|c|c|c|c|}
\hline Factor & Estrategia & Dimensión PME & $\begin{array}{l}\text { Tipología } \\
\text { de datos }\end{array}$ \\
\hline \multirow{5}{*}{ Escuela } & 1. Currículum garantizado y viable & \multirow{2}{*}{$\begin{array}{l}\text { Gestión pedagógica } \\
\text { Liderazgo formativo y académico }\end{array}$} & Proceso \\
\hline & $\begin{array}{l}\text { 2. Metas desafiantes y } \\
\text { retroalimentación efectiva }\end{array}$ & & Proceso \\
\hline & $\begin{array}{l}\text { 3. Participación de los padres } \\
\text { y la comunidad }\end{array}$ & Participación & Percepción \\
\hline & 4. Ambiente seguro y ordenado & Convivencia escolar & Percepción \\
\hline & $\begin{array}{l}\text { 5. La colegialidad y } \\
\text { la profesionalidad }\end{array}$ & No se encontró relación & Proceso \\
\hline \multirow{3}{*}{ Profesores } & 6. Estrategias de instrucción & Enseñanza y aprendizaje en el aula & Proceso \\
\hline & 7. Gestión del aula & Convivencia escolar & Percepción \\
\hline & 8. Diseño curricular del aula & Enseñanza y aprendizaje en el aula & Proceso \\
\hline \multirow{3}{*}{ Estudiantes } & 9. Ambiente familiar & Participación & Percepción \\
\hline & $\begin{array}{l}\text { 10. Incremento de habilidades } \\
\text { en lectura }\end{array}$ & Planificación y gestión de resultados & Logro \\
\hline & 11. Motivación de los estudiantes & $\begin{array}{l}\text { Apoyo al desarrollo de los } \\
\text { estudiantes }\end{array}$ & Percepción \\
\hline
\end{tabular}

Fuente: Elaboración propia (2016)

Al relacionar las estrategias de alto impacto, la tipología de datos y las dimensiones del PME se constatan importantes coincidencias. De las 11 estrategias, 10 son equivalentes en cuanto a las prácticas de gestión institucional y los tipos de datos que se generan en los procesos de monitoreo y seguimiento. Solo la estrategia de colegialidad y profesionalidad no es factible asociarla con ninguna de las dimensiones del PME. Se destaca que el área de recursos humanos y financieros del PME no encuentra equivalencia con las estrategias de alto impacto y con la tipología de datos.

Se evidencia que cinco estrategias corresponden a datos de proceso, cinco a percepción y solo una a resultados de aprendizaje. De ello se puede inferir que los datos de proceso y convivencia son relevantes para la toma de decisiones y para emprender procesos de mejora continua. Resulta también evidente que los datos sociodemográficos constituyen el telón de fondo sobre el cual se despliegan las acciones de los directores escolares.

\section{METODOLOGIA}

Esta investigación se plantea indagar sobre qué tipos de datos recolectan los directivos docentes participantes de un programa de formación de directores de excelencia y cómo se relacionan con las demandas de los PME que elaboran, implementan y evalúan anualmente. 
Este estudio se encuentra desarrollado a partir de la traducción, adaptación y validación del cuestionario de autoevaluación elaborado por Shen et al. (2012), que mide la frecuencia con que los directivos docentes recolectan datos sobre las 11 estrategias que mayor impacto tienen sobre los aprendizajes de los estudiantes (Marzano et al., 2003). El resultado se presenta en la Tabla 4:

Tabla 4. Estrategias de alto impacto en el mejoramiento escolar

\begin{tabular}{|l|l|}
\hline \multicolumn{1}{|c|}{ Factor } & \multicolumn{1}{c|}{ Estrategias (E) } \\
\hline \multirow{3}{*}{ Factor a nivel escuela } & E1. Currículum garantizado y viable. \\
& E2. Metas desafiantes y retroalimentación efectiva. \\
& E3. Participación de los padres y la comunidad. \\
& E4. Ambiente seguro y ordenado. \\
& E5. La colegialidad y la profesionalidad. \\
\hline \multirow{3}{*}{ Factor a nivel del profesor } & E6. Estrategias de instrucción. \\
& E7. Gestión del aula. \\
\hline \multirow{3}{*}{ Factor a nivel del estudiante } & E8. Diseño curricular del aula. \\
& E9. Ambiente familiar. \\
& E10. Avances en lectura. \\
& E11. Motivación de los estudiantes. \\
\hline
\end{tabular}

Fuente: Shen, Cooley, Reeves, Burt, Rainey y Yuan (2012, p. 9)

La metodología utilizada para la traducción del cuestionario de autoevaluación se desarrolló en la siguiente secuencia: se contrastaron dos traducciones al español que se realizaron de forma independiente y se generó una primera versión en español, que fue sometida a juicio de dos expertos educativos que se desempeñan profesionalmente en países de habla inglesa. Recibidas las sugerencias se desarrolló la segunda versión, la que fue analizada con el software Readability Index Calculator, el que permite medir la legibilidad (Fernández-Huerta, 1959), obteniendo 15,73 en el Nivel Flesch-Kincaid y 55 en el índice de facilidad de lectura de Flesch. Lo anterior permite concluir que es comprensible para la población que lo responderá. Luego el instrumento fue piloteado obteniendo en la prueba de alfa de Cronbach un coeficiente de 0,98 , lo que permite concluir que es fiable. El alto valor obtenido en esta prueba se puede explicar porque de las 11 estrategias, 10 son equivalentes, en cuanto a las prácticas de gestión institucional y los tipos de datos que se generan en los procesos de monitoreo y seguimiento. Por tanto, esto podría explicar la repitencia de los datos, lo que aumentaría el factor. También se realizó un análisis de validez de constructo para las 10 estrategias, en que la más baja arrojó un valor de 0,67 (E5, colegialidad y profesionalidad) y la más alta 0,91 (E4, ambiente seguro y ordenado). Lo que demuestra que el instrumento es de validez.

Cada una de estas preguntas se respondía identificando la frecuencia con que se recolectan los datos en cuatro opciones: nunca o para nada, muy poco, en cierta medida y en gran medida. En la Tabla 5 se presentan los ítems del instrumento y su relación con las estrategias de alto impacto. 
Tabla 5 Relación entre preguntas y estrategias de alto impacto.

\begin{tabular}{|c|c|}
\hline Ítem & Estrategias (E) \\
\hline 1. Realizo seguimiento e identificación y organización de los contenidos mínimos & \multirow{4}{*}{$\begin{array}{l}\text { E1. Currículum viable } \\
\text { y garantizado }\end{array}$} \\
\hline 2. Monitoreo la comunicación de las expectativas de los contenidos mínimos & \\
\hline 3. Monitoreo la distribución del tiempo para que los estudiantes aprendan los contenidos mínimos & \\
\hline 4. Monitoreo la alineación de la enseñanza en la clase con los contenidos mínimos & \\
\hline 5. Establezco metas específicas para los estudiantes de manera individual & \multirow{4}{*}{$\begin{array}{l}\text { E2. Metas desafiantes } \\
\text { y retroalimentación } \\
\text { efectiva }\end{array}$} \\
\hline $\begin{array}{l}\text { 6. Proveo una retroalimentación oportuna para el progreso de los estudiantes a través de } \\
\text { tareas alineadas de la clase }\end{array}$ & \\
\hline 7. Desarrollo metas escolares específicas y desafiantes & \\
\hline 8. Monitoreo frecuentemente el progreso de las metas escolares & \\
\hline 9. Monitoreo la frecuencia de las comunicaciones entre la escuela y los apoderados & \multirow{4}{*}{$\begin{array}{l}\text { E3. Involucramiento de } \\
\text { padres y comunidad }\end{array}$} \\
\hline 10. Monitoreo la calidad de las comunicaciones entre la escuela y los apoderados & \\
\hline $\begin{array}{l}\text { 11. Realizo un seguimiento de la participación de los apoderados y de la comunidad } \\
\text { en las actividades de la escuela }\end{array}$ & \\
\hline $\begin{array}{l}\text { 12. Realizo un seguimiento de la participación de los apoderados y de la comunidad } \\
\text { en la dirección de la escuela }\end{array}$ & \\
\hline $\begin{array}{l}\text { 13. Monitoreo la conducta de los estudiantes para establecer las expectativas de conducta } \\
\text { de toda la escuela }\end{array}$ & \multirow[t]{4}{*}{$\begin{array}{l}\text { E4. Ambiente seguro } \\
\text { y ordenado }\end{array}$} \\
\hline 14. Detecto de forma oportuna las conductas de violencia o extremas & \\
\hline $\begin{array}{l}\text { 15. Monitoreo la efectividad de las consecuencias para los estudiantes } \\
\text { con comportamientos inadecuados }\end{array}$ & \\
\hline 16. Monitoreo la evidencia de la autodisciplina y responsabilidad personal & \\
\hline 17. Realizo un seguimiento del desarrollo profesional docente & \multirow{4}{*}{$\begin{array}{l}\text { E5. Colegiabilidad } \\
\text { y profesionalismo }\end{array}$} \\
\hline 18. Monitoreo la participación de los profesores en los procesos de toma de decisiones escolares & \\
\hline 19. Realizo un seguimiento del compromiso y de la efectividad del desarrollo profesional & \\
\hline $\begin{array}{l}\text { 20. Monitoreo el desarrollo de un vocabulario profesional compartido para la enseñanza } \\
\text { y el aprendizaje }\end{array}$ & \\
\hline $\begin{array}{l}21 . \text { Monitoreo las unidades de los planes y programas para el empleo de estrategias } \\
\text { basadas en investigación }\end{array}$ & \multirow[t]{3}{*}{$\begin{array}{l}\text { E6. Estrategias } \\
\text { instruccionales }\end{array}$} \\
\hline $\begin{array}{l}\text { 22. Monitoreo la adaptación de la enseñanza en el aula para satisfacer las necesidades } \\
\text { individuales de cada estudiante }\end{array}$ & \\
\hline $\begin{array}{l}\text { 23. Monitoreo el uso que realizan los profesores de estrategias instruccionales } \\
\text { que den buenos resultados }\end{array}$ & \\
\hline 24. Monitoreo el establecimiento de reglas del aula consistentes y de rutinas de clase efectivas & \multirow[t]{3}{*}{ E7. Gestión del aula } \\
\hline $\begin{array}{l}\text { 25. Monitoreo la efectividad de las intervenciones para el comportamiento de la clase } \\
\text { con diversos estudiantes }\end{array}$ & \\
\hline 26. Monitoreo la calidad de las relaciones profesor/alumno & \\
\hline $\begin{array}{l}\text { 27. Realizo un seguimiento de la claridad que tienen los profesores respecto de objetivos } \\
\text { de la enseñanza }\end{array}$ & \multirow[t]{4}{*}{$\begin{array}{l}\text { E8. Diseño curricular } \\
\text { del aula }\end{array}$} \\
\hline 28. Monitoreo la presentación de nuevos contenidos a través de modos de aprendizaje múltiples & \\
\hline $\begin{array}{l}\text { 29. Realizo un seguimiento del uso que realizan los profesores respecto del conocimiento, } \\
\text { las habilidades y los contenidos para facilitar el aprendizaje }\end{array}$ & \\
\hline $\begin{array}{l}\text { 30. Realizo un seguimiento del involucramiento de los estudiantes en tareas más complejas } \\
\text { que involucran procesos cognitivos más altos }\end{array}$ & \\
\hline 31. Realizo un seguimiento de la comunicación de los padres con sus hijos respecto de la escuela & \multirow[t]{4}{*}{ E9. Ambiente familiar } \\
\hline 32. Realizo un seguimiento del apoyo de los padres para el aprendizaje en el ambiente familiar & \\
\hline 33. Realizo un seguimiento de evidencias de la supervisión de los padres & \\
\hline $\begin{array}{l}\text { 34. Realizo un seguimiento de las expectativas que los padres que les han comunicado } \\
\text { a sus hijos }\end{array}$ & \\
\hline 35. Realizo un seguimiento de las experiencias de los estudiantes dentro y fuera de la escuela & \multirow{4}{*}{$\begin{array}{l}\text { E10. Incremento de } \\
\text { habilidades en lectura }\end{array}$} \\
\hline $\begin{array}{l}\text { 36. Realizo un seguimiento de los patrones de lectura de los estudiantes para determinar } \\
\text { su amplitud y profundidad }\end{array}$ & \\
\hline 37. Realizo un seguimiento del desarrollo del vocabulario de los estudiantes & \\
\hline $\begin{array}{l}\text { 38. Monitoreo la enseñanza directa del vocabulario y frases que son importantes } \\
\text { para una materia específica }\end{array}$ & \\
\hline 39. Monitoreo la retroalimentación que realiza el profesor a los alumnos sobre su aprendizaje & \multirow{4}{*}{$\begin{array}{l}\text { E11. Motivación de } \\
\text { los estudiantes }\end{array}$} \\
\hline $\begin{array}{l}\text { 40. Realizo un seguimiento de las actividades de aprendizaje en función del compromiso } \\
\text { de los estudiantes }\end{array}$ & \\
\hline $\begin{array}{l}\text { 41. Realizo un seguimiento de las oportunidades de los estudiantes para construir y trabajar } \\
\text { en proyectos a largo plazo diseñados por ellos mismos }\end{array}$ & \\
\hline $\begin{array}{l}\text { 42. Realizo un seguimiento de la comprensión de los estudiantes de su motivación personal } \\
\text { y de su eficacia }\end{array}$ & \\
\hline
\end{tabular}

Fuente: Shen, Cooley, Reeves, Burt, Rainey y Yuan (2012, p. 9) 
El instrumento fue aplicado a 24 directores becarios del programa de formación de directores de excelencia, financiado por el Ministerio de Educación de Chile. Estos profesionales fueron seleccionados a través de un programa de postulación abierta en el país a partir de tres criterios: el primero corresponde a los antecedentes académicos del candidato ( $45 \%$ del total de la postulación), que se obtienen a partir del puntaje de la prueba de selección universitaria y del número de ranking de titulación de pregrado; en segundo lugar se solicitan los antecedentes profesionales (45\% del total de la postulación), compuestos por resultados de evaluación de desempeño, informe del empleador y obtención de certificaciones de calidad, por ejemplo, pertenecer a la red de maestros; finalmente, el tercer criterio de selección es un ensayo (10\%) sobre sus motivaciones para el ejercicio del cargo de director.

Este grupo (16 mujeres y 8 hombres) se caracteriza por tener un promedio de experiencia en el ejercicio del cargo de 6,7 años. De los 24 encuestados, 22 son directores de establecimientos municipales y dos de establecimientos particulares subvencionados, que se distribuyen entre 15 regiones geográficas del país. El 100\% atiende a alumnos en condiciones de pobreza y tiene un promedio de 399 estudiantes.

\section{RESULTADOS}

Para realizar el análisis se categorizó el tipo de variable y su nivel máximo de medición, considerando que se trabaja con variables cualitativas de medición ordinal y que las estrategias de alto impacto 6 y 7 cuentan con tres preguntas, y las nueve restantes, con cuatro preguntas. Se decidió llevar los datos a frecuencia relativa de porcentaje, limitándolos a una base común de 100. Por ejemplo, para el cálculo de la Estrategia 1 (Currículum garantizado y viable) se sumaron las respuestas de cada encuestado, llevándolo a porcentaje y considerando que el puntaje máximo posible de obtener es 16, si responden a cada pregunta con la opción frecuente (valor 4). De esta manera se transformaron los puntajes de una sumatoria simple a un porcentaje. Este procedimiento se realizó con cada una de las estrategias, lo que facilita la construcción de criterios comparativos. Para los factores se calculó un promedio simple de los porcentajes de cada estrategia que lo compone. Por ejemplo, para el factor Escuela se sumaron los puntajes calculados en frecuencia relativa de porcentaje correspondientes a las estrategias 1, 2, 3, 4 y 5 y se dividió por el número de estrategias que lo compone, obteniendo el resultado final.

De los datos se desprende que solo el 12,5\% $(n=3)$ de los directores declara que recoge frecuentemente información de las 11 estrategias de alto impacto. El $29,5 \%(n=7)$ recoge información de entre 8 y 6 estrategias. El 41,7\% $(n=10)$ de los encuestados recolecta información de entre 5 y 3 estrategias, y finalmente el 16,4\% $(\mathrm{n}=4)$ lo realiza solo sobre 2 o menos estrategias. Evidentemente el grupo de encuestados concentra sus resultados bajo 5 estrategias de recolección de datos. No se encontró relación entre la cantidad de estrategias en que se recolecta información frecuentemente y el número de alumnos de la escuela. Lo mismo sucede con los años de experiencia en el cargo. 
Si el análisis se realiza por factores Escuela, Profesores y Estudiantes, estos últimos tienen los porcentajes más bajos: solo una de las tres estrategias que lo componen, E10 (Incremento de habilidades de lectura), supera el 60\% de frecuencia de recolección. Para el factor Profesor, compuesto por tres estrategias, solo E7 (Gestión de aula) obtiene una alta frecuencia de recolección de datos, con un 83,6\%. Finalmente, el factor Escuela, compuesto por 5 estrategias (ver Tabla 3), es el que reúne el mayor número de frecuencia de recolección de datos, encontrándose E4 (Ambiente seguro y ordenado) con un 83\% y E3 (Participación de los padres y la comunidad) con $75 \%$ de las respuestas seleccionadas como frecuentemente.

Para profundizar el análisis de datos se elaboró un ranking agrupando las respuestas a las preguntas valoradas como frecuentemente y se asoció a estas el tipo de dato, como se muestra en la Tabla 6.

Tabla 6. Ranking de estrategias de alto impacto versus factor $y$ tipo de datos

\begin{tabular}{|c|l|c|c|c|}
\hline \multirow{2}{*}{ Ranking } & \multicolumn{1}{|c|}{ Estrategia } & \multicolumn{1}{c|}{$\begin{array}{c}\text { \% de } \\
\text { respuestas } \\
\text { frecuentemente }\end{array}$} & \multicolumn{1}{|c|}{$\begin{array}{c}\text { Factor } \\
\text { Tipo de } \\
\text { dato }\end{array}$} \\
\hline \multirow{2}{*}{1} & E7. Gestión del aula. & 83,3 & Profesor & Percepción \\
\cline { 2 - 5 } & E4. Ambiente seguro y ordenado. & 83,3 & Escuela & Percepción \\
\hline \multirow{2}{*}{2} & E3. Participación de los padres y la comunidad. & 75,0 & Escuela & Percepción \\
\hline \multirow{2}{*}{3} & E10. Incremento de habilidades en lectura. & 62,5 & Estudiante & Logro \\
\hline \multirow{2}{*}{4} & E1. Currículum garantizado y viable. & 58,3 & Escuela & Proceso \\
\cline { 2 - 5 } & E5. La colegialidad y la profesionalidad. & 50,0 & Escuela & Proceso \\
\cline { 2 - 5 } & E8. Diseño curricular del aula. & 50,0 & Profesor & Proceso \\
\hline \multirow{2}{*}{5} & E2. Metas desafiantes y retroalimentación efectiva. & 29,2 & Escuela & Proceso \\
\cline { 2 - 6 } & E6. Estrategias de instrucción. & 29,2 & Profesor & Proceso \\
\cline { 2 - 5 } & E9. Ambiente familiar para el aprendizaje. & 29,2 & Estudiante & Percepción \\
\cline { 2 - 5 } & E11. Motivación de los estudiantes. & 29,2 & Estudiante & Percepción \\
\hline
\end{tabular}

Fuente: Elaboración propia (2016)

El ranking elaborado consta de cinco niveles. El primero está compuesto por dos estrategias: E7 (Gestión del aula, 83,3\%) y E4 (Ambiente seguro y ordenado, 83,3\%), ambas asociadas al comportamiento de los alumnos, es decir, a la dimensión convivencia escolar del PME. En el segundo nivel de ranking se encuentra solo una estrategia: E3 (Participación de los padres y la comunidad, 75\%). El tercer lugar lo ocupa la E10 (Incremento de las habilidades de lectura, 62\%). En el cuarto lugar se ubican tres estrategias: E1 (Currículum garantizado y viable, 58,3\%), E5 (La colegialidad y la profesionalidad, 50\%) y E8 (Diseño curricular del aula, 50\%). Y finalmente en el quinto lugar del ranking se encuentran cinco estrategias: E2 (Metas desafiantes y retroalimentación efectiva, 29,2\%), E6 (Estrategias de instrucción, 29,2\%), E9 (Ambiente familiar para el aprendizaje, 29,2\%) y E11 (Motivación de los estudiantes, $29,2 \%)$.

De lo anterior se deduce que los directores fundamentalmente están interesados en recolectar datos sobre convivencia escolar, luego sobre cobertura curricular y finalmente sobre prácticas de aula. Desde la perspectiva de la tipología 
de datos, están interesados en primer lugar en datos de percepción, en segundo lugar en los logros (resultados) y en tercer lugar en procesos de enseñanza y aprendizaje. Estos intereses se asocian al ejercicio de actividades de gestión de la convivencia escolar y control curricular, más que al liderazgo pedagógico. Lo anterior implica que los directivos docentes no cuentan con información sobre las prácticas de enseñanza que implementan los profesores, dificultando la posibilidad de realizar un análisis de datos múltiples, por ejemplo, efectuar un cruce de datos entre procesos de aula con percepciones de los estudiantes.

Tomar decisiones para elaborar un PME en base al cruce de dos tipos de datos, percepción y logro, implica que el énfasis de la gestión del director se apoya en las opiniones y creencias sobre el comportamiento de los alumnos y en los resultados de aprendizaje, que son datos interesantes, porque demuestran el estado de situación; sin embargo, al no estar relacionados con datos de proceso, que informen sobre qué se hace en la aula, impiden realizar un análisis que identifique las causas raíces de los problemas.

El diagnóstico en los planes de mejoramiento y el involucramiento de la comunidad necesitan datos de diferente tipo. A su vez la realización de cruces entre ellos y la formulación de preguntas desafiantes potencian una aproximación más efectiva a la naturaleza del problema identificado (Bernhardt, 2003). De tal forma de animar a la comunidad escolar a encontrar nuevas explicaciones a sus problemas o a consensuar que algunos son síntomas y que los problemas son otros, no identificados anteriormente. Los datos de proceso permiten analizar las prácticas pedagógicas e institucionales y desarrollar respuestas novedosas que alivianen la carga que significa emprender cambios (Argyris y Shön, 1978).

\section{DISCUSIÓN Y CONCLUSIONES}

A partir de los resultados del estudio es posible observar que los directores están muy interesados en recoger información sobre clima y convivencia escolar (Datos de percepción), con la finalidad de identificar casos de alumnos disruptivos. En segundo término, los directores demuestran interés por monitorear la comunicación entre la escuela y los padres (Datos de percepción) y los logros académicos de los estudiantes en lectura (Dato de resultado) y, en tercer lugar, monitorear las estrategias de instrucción (Datos de proceso). Esta secuencia de recogida de datos presenta un orden de importancia donde predominan los datos de percepción.

Contar con datos de pocas estrategias implica por lo menos dos cuestiones preocupantes cuando las escuelas quieren mejorar. La primera de ellas es diseñar acciones en los PME en forma intuitiva sin lograr identificar los nudos críticos de los procesos de enseñanza y aprendizaje que son necesarios para mejorar, y la segunda es implementar acciones que no logren los resultados esperados, lo que decepcionará a los involucrados en su realización y a la comunidad. Estos dos aspectos, conjugados en comunidades que atienden a estudiantes en contextos de pobreza, pueden implicar que la escuela quede inmovilizada. Utilizando el concepto 
de Harris y Chapman (2004), en las escuelas donde se observa baja colaboración entre los docentes y su accionar depende de la rendición de cuentas externas sin rendición de cuentas internas son más proclives a contar con ausencia de datos de proceso. Es decir, los datos de procesos sistematizados y transformados en información pueden animar la reflexión de los profesores y movilizar cambios en las prácticas. El no contar con este tipo de datos dificulta la labor directiva (Elmore, 2010).

De acuerdo a las categorías de prácticas analizadas en el marco teórico, es factible señalar que los directivos docentes de este estudio no realizan acciones de gestión de la instrucción y monitoreo de la enseñanza, por lo que su liderazgo no se orienta al aprendizaje sino al control de la disciplina escolar a nivel institucional y de aula, fomentando el involucramiento de los padres en las actividades de la escuela (por ejemplo, celebraciones de festividades) y no en el apoyo a los procesos de aprendizaje de sus hijos.

Desde la perspectiva de las dimensiones del PME se puede evidenciar que la mayor parte de las acciones que cuentan con datos son las de convivencia escolar. Esta se convierte en la base del aprendizaje. El supuesto que subyace es que logrando la normalización en el comportamiento de los alumnos es factible implementar actividades de aprendizaje en el aula. Este se contrapone al que postula que una didáctica adecuada promueve la motivación y el compromiso de los estudiantes y por lo tanto el buen comportamiento. De ahí que para los directores sea una preocupación constante la normalización como un paso previo y necesario para instalar procesos de mejora pedagógica (Harris, 2009; Harris y Chapman, 2004).

Lo anterior evidencia que administración, gestión y liderazgo son conceptos que parecen estar constantemente superpuestos entre sí. Implícitamente se advierte que control, orden y disciplina funcionan como principios esenciales para los directores encuestados. De modo tal que para estos directores escolares liderar se convierte en un proceso de control más que de influencia. Esto se encuentra en coherencia con la visión de que el resultado de aprendizaje de los alumnos se vincula directamente con el compromiso de los padres, sin considerar al profesor y sus prácticas pedagógicas como el principal agente de cambio dentro de la escuela.

Finalmente se hace explícito que el cuestionario traducido y adaptado arroja resultados interesantes y que puede ser utilizado como diagnóstico en procesos formativos de directivos escolares. Como se evidenció en el marco teórico, el uso de datos por directivos y profesores cuenta con barreras formativas y de profesionalidad, por lo que para estimular su uso en contextos de rendiciones de cuentas externas y de implementación de planes de mejora requiere de acompañamiento. Un ejemplo es el programa documentado por Lynch, Smith, Provost y Madden (2016) que describe un modelo de reforma escolar enfocado a través de una combinación de liderazgo distribuido y toma de decisiones basadas en datos, dentro de un contexto de entrenamiento, tutoría y retroalimentación.

Un aspecto que no es discutido en este estudio es la oportunidad en que se recolectan, procesan y se utilizan los datos. Como plantea Elmore (2010), para apoyar un proceso de toma de decisiones consciente e intencional es necesario recolectar 
datos en "tiempo real", que interconecten decisiones pedagógicas con acciones basadas en evidencias, actividad muy importante en la implementación de planes de mejora, con el objeto de monitorear los resultados de las acciones realizadas. Esto con la finalidad de influenciar las prácticas docentes a partir de evidencia y poder enjuiciar las decisiones que se han tomado.

\section{REFERENCIAS}

Anderson, L., y Bennet, N. (2003). Developing educational leadership. Londres, Inglaterra: SAGE.

Argyris, C., y Shön, D. A. (1978). Organizational Learning: A theory of action perspective. Reading, MA: Addison Wesley.

Bernhardt, V. (2003). Using data to improve student learning in elementary schools. Nueva York, NY: Eye Education.

Bolívar, A. (2012). Políticas actuales de mejora y liderazgo educativo. Madrid, España: Ediciones Aljibe. Canto-Mayo, I. (2004). Planes de mejora en los centros educativos. Málaga, España: Ediciones Aljibe.

Cooley, V. E., Shen, J., Miller, D., Winograd, P. N., Rainey, J. M., Yuan, W., y Ryan, L. (2006). Increasing leaders' capacity in data-based decision-making: State-level initiatives in Ohio, New Mexico, and Michigan. Educational Horizons, 85, 57-64.

Datnow, A., y Hubbard, L. (2016). Teacher capacity for and beliefs about data-driven decision making: A literature review of international research. Journal of Educational Change, 17(1), 7-28.

Drucker, P. F. (2002). La gerencia en la sociedad futura. Bogotá, Colombia: Editorial Norma.

Elmore, R. (2010). Mejorando la escuela desde la sala de clases. Santiago, Chile: Fundación Chile.

Fernández-Huerta, J. (1959). Medidas sencillas de lecturabilidad. Consigna, 214, $29-32$.

Fullan, M. (2007). The new meaning of educational change. Nueva York, NY: Routledge.

Fullan, M. (2009). Leadership development the larger context. Educational leadership, 67(2), 45-49.

Hargreaves, A., y Fink, D. (2006). Sustainable Leadership. San Francisco, CA: Jossey-Bass Education Series.

Harris, A. (2009). Distributed Leadership, Different Perspectives. Londres, Inglaterra: Springer.

Harris, A., y Chapman, C. (2004). Improving schools in difficult contexts: Towards a differentiated approach. British Journal of Educational Studies, 52(4), 417-431.

Heifetz, R. (1994). Leadership without easy answers. Boston, MA: Harvard University Press.

Honig, M. I., y Coburn, C. (2008). Evidence-based decision making in school district central offices: Toward a policy and research agenda. Educational Policy, 22(4), 578-608.

Knapp, M. S., Swinnerton, J. A., Copland, M. A., y Monpas-Huber, J. (2006). Data-informed leadership in education. Seattle, WA: Center for the Study of Teaching and Policy, University of Washington.

Leithwood, K., y Riehl, C. (2005). What do we already know about educational leadership?. En W. Firestone y C. Riehl (Eds.), A New Agenda for Research in Educational Leadership (pp. 12 27). Nueva York, NY: Teachers College Press.

Leithwood, K., Harris, A., y Hopkins, D. (2008). Seven strong claims about successful school leadership. School leadership and management, 28(1), 27-42. 
Lynch, D., Smith, R., Provost, S. y Madden, J. (2016). Improving teaching capacity to increase student achievement: the key role of data interpretation by school leaders. Journal of Educational Administration, 54(5), 575-592.

Marsh, J. A., y Farrell, C. C. (2015). How leaders can support teachers with data-driven decision making: A framework for understanding capacity building. Educational Management Administration \& Leadership, 43(2), 269-289.

Marzano, R. J., Waters, T., y McNulty, B. A. (2003). What works in schools. Alexandria, VA: Association of Supervision and Curriculum Development.

Ministerio de Educación de Chile. (2018). Orientaciones técnicas para sostenedores y directivos escolares. Plan de Mejoramiento Educativo. Nuevo enfoque a 4 años. Recuperado de https://www.mineduc.cl/wp-content/uploads/sites/19/2018/02/PME-2018-Orientaciones-27feb.pdf

Ministerio de Educación de Ecuador. (2012). Orientaciones para la elaboración de Planes de Mejora. Recuperado de https://educacion.gob.ec/wp-content/uploads/downloads/2012/08/Plan_Mejora1.pdf

Ministerio de Educación de Perú. (2013). Manual de elaboración, costeo y presupuesto de Planes de Mejora. Proceso de Mejora de la Gestión Escolar, camino a la Acreditación. Recuperado de: http://www.fondep.gob.pe/wp-content/uploads/2014/01/Manual.pdf

Programa de las Naciones Unidas para el Desarrollo. (2009). La manera de hacer las cosas. Recuperado de http://hdr.undp.org/sites/default/files/chile-2009-sinopsis.pdf

Robinson, V., Lloyd, C., y Rowe, K. (2008). The impact of leadership on student outcomes: An analysis of the differential effects of leadership types. Educational Administration Quarterly 44(5), 564 588.

Schildkamp, K., y Kuiper, W. (2010). Data-informed curriculum reform: Which data, what purposes, and promoting and hindering factors. Teaching and teacher education, 26(3), 482-496.

Seyal, A. H. (2015). Examining the role of transformational leadership in technology adoption: Evidence from Bruneian technical \& vocational establishments (TVE). Journal of Education and Practice, 6(8), 32-43.

Shen, J., Berry, J., Cooley, V., Kirby, B., Marx, G., y Whale, D. (2007). Data-informed decision-making: A guidebook for data points and analyses in the context of Michigan School Improvement Framework. Kalamazoo, MI: Michigan Coalition of Educational Leadership.

Shen, J., y Cooley, V. (2008). Critical issues in using data for decision-making. International Journal of Leadership in Education, 11, 319-329.

Shen, J., Cooley, V. E., Ma, X., Reeves, P. L., Burt, W. L., Rainey, J. M., y Yuan, W. (2012). Datainformed decision making on high-impact strategies: Developing and validating an instrument for principals. The Journal of Experimental Education, 80(1), 1-25.

Swaffield, S., y Macbeath, J. (abril de 2009). Researching leadership for learning across international and methodological boundaries. Paper presented at annual meeting of the AERA, San Diego, CA. 